\title{
Responsabilidad Social y Desarrollo Sustentable de la ciudad de Pilar; Una mirada desde la percepción de los estudiantes universitarios
}

\section{RESUMEN}

Edgar Daniel Morell Jiménez

edjm_25@hotmail.com

Andrés Abelino Villalba Chamorro

anvill65@hotmail.com

Universidad Nacional de Misiones

La responsabilidad social busca "el desarrollo sostenible, tanto social como ambiental, de la sociedad y no solamente una responsabilidad reactiva (no causar daño, portarse bien), sino proactiva (participar, innovar, mejorar), donde la participación en el bien común es un deber de la responsabilidad social" (Vallaeys \& Álvarez Rodríguez, 2019, pág. 97). El presente estudio muestra la percepción de los estudiantes universitarios sobre la Responsabilidad Social y Desarrollo Sustentable de la ciudad de Pilar, en representación del Municipio, la disposición y administración de sus bienes e ingresos y la prestación de los servicios públicos en general. Se analizaron variables relacionadas a la propuesta de Wong (2005), que comprende; a) estructura económica, agentes económicos e innovación, b) Infraestructura, servicios y accesibilidad, c) Calidad de vida y RRHH, d) Cohesión social, organizaciones e identidad territorial, e) Medio ambiente y Recursos Naturales y f) Organizaciones, Normatividad y Programas de ordenamiento ecológico y territorial. Se adoptó una metodología descriptiva, no experimental, trasversal prospectivo con enfoque únicamente cuantitativo (Hernandez Sampieri, Fernandez Collado, \& Pilar Batista, 2014). Como instrumento de recolección de datos se utilizó el cuestionario "Percepción sobre el nivel de desarrollo sustentable, desde la perspectiva del sector educativo" elaborado y validado por Galván, Miranda, Báez, Acosta, \& Murillo (2014). La población está conformada por 130 estudiantes universitarios matriculados en el año 2020, en la carrera de licenciatura en Contaduría Publico Nacional, dependiente de la Facultad de Ciencias Contables, Administrativas y Económicas de la Universidad Nacional de Pilar, Ñeembucú, Paraguay. Los principales hallazgos muestran que desarrolla un nivel intermedio de Responsabilidad Social y Desarrollo Sustentable, desde la percepción de los estudiantes universitarios. Dicho nivel se traduce en acciones mínimas aceptables de desarrollo, por tal razón, el panorama actual de la ciudad de Pilar, desde las primeras impresiones de los estudiantes universitarios, motiva a abordar mejoras en cuanto a las seis dimensiones que propone Wong (2005) estructura económica, agentes económicos e innovación, Infraestructura, servicios y accesibilidad, Calidad de vida y RRHH, Cohesión social, organizaciones e identidad territorial, Medio ambiente y Recursos Naturales y Organizaciones, Normatividad y Programas de ordenamiento ecológico y territorial, ya que en ninguna de las dimensiones citadas se han evidenciado indicadores en condiciones de fortalezas.

Palabras Clave: Responsabilidad Social, Desarrollo Sustentable, Municipio. 


\title{
Social Responsibility and Sustainable Development of the city of Pilar; A look from the perception of university students
}

\begin{abstract}
ABSTRAC: Social responsibility seeks "the sustainable development, both social and environmental, of society and not only reactive responsibility (do not cause harm, behave well), but also proactive (participate, innovate, improve), where participation in good common is a duty of social responsibility" (Vallaeys \& Álvarez Rodríguez, 2019, p. 97). This study shows the perception of university students about Social Responsibility and Sustainable Development of the city of Pilar, on behalf of the Municipality, the disposition and administration of its assets and income and the provision of public services in general. Variables related to the proposal of Wong (2005) were analyzed, which includes; a) Economic structure, economic agents and innovation, b) Infrastructure, services and accessibility, c) Quality of life and HR, d) Social cohesion, organizations and territorial identity, e) Environment and Natural Resources and f) Organizations, Regulations and Programs of ecological and territorial ordering. A descriptive, non-experimental, prospective cross-sectional methodology was adopted with a solely quantitative approach (Hernandez Sampieri, Fernandez Collado, \& Pilar Batista, 2014). As a data collection instrument, the questionnaire "Perception of the level of sustainable development, from the perspective of the educational sector" was used, prepared and validated by Galván, Miranda, Baéz, Acosta, \& Murillo (2014). The population is made up of 130 university students enrolled in 2020, in the undergraduate degree in National Public Accounting, dependent on the Faculty of Accounting, Administrative and Economic Sciences of the National University of Pilar, Neembucú, Paraguay. The main findings show that it develops an intermediate level of Social Responsibility and Sustainable Development, from the perception of university students. This level is translated into minimum acceptable development actions, for this reason, the current panorama of the city of Pilar, from the first impressions of university students, motivates to address improvements in terms of the six dimensions proposed by Wong (2005) structure economic, economic agents and innovation, Infrastructure, services and accessibility, Quality of life and HR, Social cohesion, organizations and territorial identity, Environment and Natural Resources and Organizations, Regulations and ecological and territorial planning programs, since in none of the cited dimensions indicators have been evidenced in conditions of strengths.
\end{abstract}

Keywords: Social Responsibility, Sustainable Development, Municipality

Artículo recibido: 19. agosto. 2020 Aceptado para publicación: 12. setiembre. 2020 Correspondencia: edjm_25@hotmail.com Conflictos de Interés: Ninguna que declarar 


\section{INTRODUCCIÓN}

La investigación aborda sobre la Responsabilidad Social y el Desarrollo Sustentable de la ciudad de Pilar desde la percepción de un grupo de estudiantes universitarios, entendiéndose como responsabilidad social a la reflexión sobre el desempeño del municipio de Pilar-Ñeembucú, Paraguay y su impacto en la sociedad, también como una obligación social o filantrópica, como estrategia proactiva de dialogada con los grupos de interés, promoviendo la honestidad y responsabilidad social en los involucrados en la sociedad (López Regalado \& Ahumada Tello, 2018).

La responsabilidad social busca "el desarrollo sostenible, tanto social como ambiental, de la sociedad y no solamente una responsabilidad reactiva (no causar daño, portarse bien), sino proactiva (participar, innovar, mejorar), donde la participación en el bien común es un deber de la responsabilidad social” (Vallaeys \& Álvarez Rodríguez, 2019, pág. 97).

Pero, la responsabilidad social no solo la gestiona un ente, si no concierne a todas las organizaciones, que de alguna manera provocan un impacto en la sociedad y en el medio ambiente, es por ello que deben de contribuir a un desarrollo social y ambiental sostenible, que no tiene como principal protagonista a la empresa, si no también, trata de todos los actores sociales desde sus respectivos objetivos y quehacer institucional para buscar un bien común en el cual involucrarse y beneficie a todas las partes (Vallaeys \& Álvarez Rodríguez, 2019).

Investigaciones recientes, como la desarrollada por Parra Melgarejo (2018) sobre la Percepción de la responsabilidad social empresarial por parte de los trabajadores municipales concluyeron que la percepción en los trabajadores es moderada, ello quiere decir que los empleados conocen sobre el tema y tratan de poner en práctica en sus diferentes áreas laborales, pero aún falta la implementación y adopción total de la responsabilidad social.

Otro estudio desarrollado por Gallardo-Milanés, Satiê de Olivera-Pátaro, \& Mezzomo, (2019) sobre el estudiantado universitario de UNESPAR-Brasil: sus percepciones sobre el desarrollo sostenible. El estudio reveló que los estudiantes participan poco en actividades ecológico- ambientales a pesar de reconocer la importancia del medioambiente y poseer un juicio crítico al respecto, muestran saber los problemas 
ambientales globales y locales sin embargo no revelaron una clara percepción sobre la condición ecosistémica y compleja del mundo, por lo que son limitadas sus ideas sobre desarrollo sostenible. Estudio similar elaborado por Palomino-Garcia (2020) sobre Responsabilidad social, concluyo que no se les provee oportunidades a los estudiantes universitarios para involucrarse en actividades socialmente responsables.

En Paraguay los estudios sobre Responsabilidad Social y Desarrollo sostenible son escasos, siendo lo más citados aquellos desarrolladas dentro el ámbito universitario (Fonseca Ferís, 2020, Barrios \& Nuñez Esquivel, 2019, Coppari, Bagnoli, \& Codas, 2016, Perez Colina, 2020) y por la Asociación de Empresarios Cristianos (ADEC).

Gracias a la ISO 26000, la responsabilidad social (RS) está ahora bien definida: (1) $\mathrm{Su}$ contenido es la gestión de los impactos (directos e indirectos) de la organización, tanto hacia dentro como hacia fuera de sí misma; (2) Su fin es el desarrollo humano y la sostenibilidad social y ambiental de la comunidad humana; (3) Su medio es la participación de todas las partes interesadas que pueden influir en el desarrollo sostenible, conforme a las leyes y normas internacionales de comportamiento. (Vallaeys \& Álvarez Rodríguez, 2019, pág. 98).

Para Gallardo-Milanés, Olivera-Pátaro, \& Mezzomo (2019) los países latinoamericanos, entre ellos Paraguay, enfrentan el desafío de gestionar adecuadamente su ambiente, donde los jóvenes juegan un papel significativo y son un elemento social clave para influir en el debate público buscando involucrarse con la responsabilidad social y el desarrollo sustentable de su entorno.

Actualmente, hablar de desarrollo sostenible abre un debate en torno a su cumplimiento, ya que la sociedad actual enfrenta dilemas por lo complejo que significa interrelacionar lo natural, lo social, cultural, tecnológico, institucional y lo económico (Gómez y GómezSal, 2013). No obstante, es imprescindible su abordaje, ante el actual contexto en el que predomina el uso indiscriminado de los recursos naturales, haciendo énfasis en la sustentabilidad como principal alternativa para el progreso (Gallardo-Milanés, OliveraPátaro, \& Mezzomo, 2019). 
Se entiende como «desarrollo sostenible» a las "práctica que incluye el impulso de movimientos sociales, la organización de las instituciones, la elaboración de la ciencia y la tecnología y la negociación de compromisos entre quienes se preocupan por el medio ambiente, la economía y los aspectos sociales" (Robert, Parris, \& Leiserowitz, 2005, como se cita en Rodrigo-Cano, Picó, \& Dimuro, 2019, pág. 26).

Para fomentar la adopción de la Responsabilidad Social y el Desarrollo Sustentable, la Naciones Unidas establece 17 objetivos denominados Desarrollo Sostenible (ODS), en su Agenda 2030, haciendo que todos los países miembros, y entre ellas Paraguay, se comprometa mediante la implementación dentro de su plan de gestión, hacer énfasis en el cuidado del medio ambiente a través de un cambio de estilo de vida para garantizar la continuidad de los recursos naturales (ONU Organización de la Naciones Unidas, 2016).

El desarrollo sustentable se fundamenta en principios éticos, como el respeto al proceso de regeneración de los ciclos naturales, valores políticos, como la democracia participativa y equidad social, y normas de comportamiento y conductas morales, como la racionalidad ambiental. El desarrollo sustentable es descentralizado y autogestionario, capaz de satisfacer las necesidades básicas de la población, respetar la diversidad cultural y mejorar la calidad de vida, sin degradar el ciclo natural. (Martínez Castillo \& Martínez Chaves, 2016, pág. 123).

\section{Desarrollo Local Responsable y Sustentable}

No existe una única definición de Desarrollo Local, no obstante, la más utilizada es la que se define como un proceso de transformación de la economía y de la sociedad orientada al uso de sus recursos internos para desarrollar un entorno institucional, político y cultural de fomento de las actividades productivas y de generación de empleo en los diferentes ámbitos territoriales (Lopez Paniagua, Ayala Ortiz, \& Arellanes Cancino, 2017).

"El enfoque del desarrollo local ubica en el centro del proceso a los actores sociales y a una serie de factores locales que impulsan el desempeño económico, social y político, y propone, aunque no explícitamente, un aprovechamiento racional de los recursos naturales" (López y Chauca 2010, como se cita en Lopez Paniagua, et.al, 2017). 
No obstante, Hernández (2013) señala que actualmente se evidencian multiples conductas irresponsables con ausencia de ética, tanto en las empresas como en la administración pública, pero que aun así, la Responsabilidad Social puede convertirse en una herramienta que puede aportar nuevas alternativas al desarrollo local para escapar de la crisis a través del fomento de la integración y de las colaboraciones empresas-administraciones públicas (como se cita en Pérez-García, 2017, p. 188). Pero este compromiso, solo será efectivo cuando "las empresas y administraciones públicas tomen decisiones basadas en la sostenibilidad desde un planteamiento integrado y estratégico atendiendo a la demanda de los grupos de interés y no como una estrategia de maquillaje de imagen corporativa" (Pérez-García, 2017, pág. 189).

Poner en marcha y dar continuidad a un proceso de desarrollo participativo requiere una esfera publica donde se encuentren todas las visiones, identidades y voluntades, donde se diriman las pretensiones de legitimidad de los intereses particulares y se llegue a acuerdos que permitan movilizar todas las capacidades con sinergia. Cuando el sistema partidario y de gobierno dominantes evitan abrir espacios públicos que no puedan controlar y manipular, la universidad puede contribuir a ofrecer un espacio público pluralista, convocando a todos los sectores a tratar de manera transparente los problemas de la sociedad local en el contexto nacional y mundial. (Coraggio, 2002, pág. 14).

Wong (2005) propone una estructura para el análisis estratégico de desarrollo sustentable, basada en cuatro ámbitos fundamentales del desarrollo local-regional que se puede observar en la ilustración 1. 


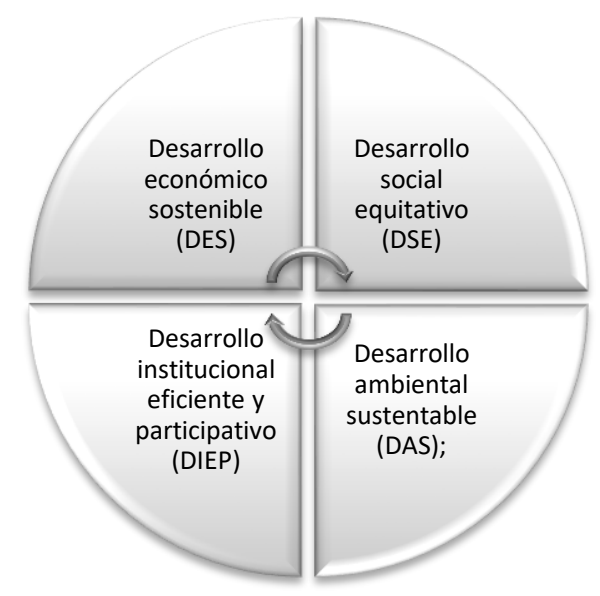

Ilustración 1. Análisis Estratégico de DS de Wong (2005).

Entendiendo la importancia, no solo de desarrollar acciones responsables y sustentables en lo social, económico y ambiental, si no también, trasmitir dichos valores a las personas, y más a la población joven, para incluirlas en la creación de un estila de vida que se adecue y responda a los objetivos del desarrollo sostenible, se desarrolla la investigación cuyo objetivo busca conocer la percepción de los estudiantes universitarios sobre las acciones de RS y DS en la ciudad de Pilar.

\section{METODOLOGÍA}

La investigación adopta una metodología de tipo descriptivo con enfoque cuantitativo, trasversal, prospectivo (Hernandez Sampieri, Fernandez Collado, \& Pilar Batista , 2014), con el fin de conocer la percepción de los estudiantes de la carrera de Contaduría Público Nacional, sobre la Responsabilidad Social y Desarrollo Sustentable de la ciudad de Pilar.

La población está conformada por 130 estudiantes universitarios matriculados en el año 2020, en la carrera de licenciatura en Contaduría Publico Nacional, dependiente de la Facultad de Ciencias Contables, Administrativas y Económicas de la Universidad Nacional de Pilar, Neembucú, Paraguay. La muestra está representada por el total de la población, ya que es accesible a los investigadores, por el tamaño reducido del grupo de estudio. Por tal razón, no se emplea técnicas de selección de muestras.

Como instrumento de recolección de datos se utilizó el cuestionario "Percepción sobre el nivel de desarrollo sustentable, desde la perspectiva del sector educativo" elaborado y 
validado por Galván, Miranda, Baéz, Acosta, \& Murillo (2014). El cuestionario posee una escala tipo Likert, con seis valores posibles de respuestas (nulo/inexistente; muy bajo; bajo; medio/mínimo aceptable; alto y muy alto). Categorizadas por 6 dimensiones como; 1) estructura económica, agentes económicos e innovación, 2) Infraestructura, servicios y accesibilidad, 3) Calidad de vida y RRHH, 4) Cohesión social, organizaciones e identidad territorial, 5) Medio ambiente y Recursos Naturales y 6) Organizaciones, Normatividad y Programas de ordenamiento ecológico y territorial.

El cuestionario se aplicó de manera online a través del formulario Google forms, como forma de respetar las medidas de aislamiento social por pandemia Covid-19. Una vez recolectado la información se procedió al procesamiento del mismo, utilizando el software de Microsoft Excel para matriz de datos, utilizando para su representación tablas de datos porcentuales, posterior a ello se realizó el análisis y la discusión.

Como criterios de inclusión; se eligió, como parte del estudio, a los estudiantes de la carrera de Contaduría Publico Nacional, por ser alumnos de los investigadores, que al mismo tiempo son docentes, y porque se encuentran desarrollando contenidos curriculares relacionadas a la temática de estudio. Se excluyó a alumnos de otras carreras, sedes y/o egresados, por no tener contenidos en común con los investigadores.

La participación de los alumnos fue voluntaria y anónima, respetando, en cada pregunta, mantener la confidencialidad de los estudiantes.

\section{RESULTADOS}

En cuanto a la dimensión Estructura económica, agentes económicos e innovación; se puede observar en la tabla 1 que los estudiantes percibieron que en los últimos cinco años se han manifestado niveles aceptables a altos de inversiones, crecimiento de negocios locales, entre otras en la ciudad de Pilar, pese a que percibieron bajos a muy bajos índices de implementación de programas de apoyo a las empresas. Así mismo, perciben, en un nivel medio aceptable, las contribuciones al desarrollo social de la ciudad, por parte de las MiPymes. Al mismo tiempo de manifestar niveles bajos referentes a informaciones sobre programas que ofrezcan apoyos a MiPymes en la ciudad. No obstante, en un nivel alto consideran la idea de invertir en la ciudad o innovar algo ya existente. 
Por otro lado, los estudiantes perciben deficiente niveles en componentes como: asociaciones a la unión entre los empresarios-comerciantes de la ciudad, al acceso y promoción de nuevas tecnologías para desarrollar una empresa y condiciones ambientales-laborales para establecer negocios, al mismo tiempo, consideran que mínimamente se provechan los recursos locales (humanos, naturales) en el desempeño de actividades productivas. Elementos que están directamente relacionadas con la responsabilidad social y el desarrollo sustentable (Fernández \& Gutiérrez , 2013).

Tabla 1. Estructura Económica, agentes económicos e innovación.

\begin{tabular}{|c|c|c|c|c|c|c|}
\hline & $\begin{array}{l}\text { Nulo/ } \\
\text { inexistente }\end{array}$ & $\begin{array}{l}\text { Muy } \\
\text { Bajo }\end{array}$ & Bajo & $\begin{array}{l}\text { Medio/ } \\
\text { mínimo } \\
\text { aceptable }\end{array}$ & Alto & $\begin{array}{l}\text { Muy } \\
\text { Alto }\end{array}$ \\
\hline $\begin{array}{l}\text { Últimos } 5 \text { años se han manifestado } \\
\text { inversiones, crecimiento de negocios } \\
\text { locales, entre otras en la ciudad }\end{array}$ & 3,6 & 15,3 & 13,1 & 43,8 & 22,6 & 1,5 \\
\hline $\begin{array}{l}\text { Existe programas de apoyos a empresas en } \\
\text { la ciudad }\end{array}$ & 11,8 & 27,9 & 35,3 & 23,5 & 0,7 & 0,7 \\
\hline $\begin{array}{l}\text { Las MiPymes contribuyen al desarrollo } \\
\text { social de la comunidad a través de acciones } \\
\text { sociales }\end{array}$ & 2,9 & 10,3 & 23,5 & 40,4 & 20,6 & 2,2 \\
\hline $\begin{array}{l}\text { Información sobre programas para } \\
\text { pequeñas y medianas empresas }\end{array}$ & 16,2 & 19,9 & 30,9 & 28,7 & 3,7 & 0,7 \\
\hline $\begin{array}{l}\text { En esta ciudad, te gustaría o piensas en } \\
\text { iniciar un negocio }\end{array}$ & 1,5 & 1,5 & 5,8 & 35 & 46 & 10,2 \\
\hline $\begin{array}{l}\text { Te interesa la idea de crear algo o de } \\
\text { hacerle una innovación de algo que ya } \\
\text { existe }\end{array}$ & 0,7 & 1,5 & 4,4 & 17,5 & 53,3 & 22,6 \\
\hline $\begin{array}{l}\text { Unión entre los empresarios-comerciantes } \\
\text { de la ciudad }\end{array}$ & 5,1 & 14,6 & 27,7 & 40,1 & 12,4 & 0 \\
\hline $\begin{array}{l}\text { Acceso y promoción de nuevas tecnologías } \\
\text { para desarrollar una empresa }\end{array}$ & 6,6 & 13,1 & 25,5 & 32,1 & 19,7 & 2,9 \\
\hline $\begin{array}{l}\text { Condiciones ambientales y laborales para } \\
\text { establecer negocios }\end{array}$ & 0,7 & 10,9 & 23,4 & 35,8 & 27,7 & 1,5 \\
\hline $\begin{array}{l}\text { Se provechan los recursos locales } \\
\text { (humanos, naturales) en el desempeño de } \\
\text { actividades productivas }\end{array}$ & 2,9 & 7,3 & 20,4 & 47,4 & 19 & 2,9 \\
\hline Son buenos los salarios en la ciudad & 5,9 & 43,4 & 25,7 & 23,5 & 1,5 & 5,9 \\
\hline $\begin{array}{l}\text { Existen fuentes de financiamiento para } \\
\text { MiPymes en la ciudad }\end{array}$ & 5,8 & 13,9 & 36,5 & 31,4 & 12,4 & 0 \\
\hline
\end{tabular}

Fuente: elaboración propia

En cuanto a la segunda dimensión Infraestructura, servicios y accesibilidad; los estudiantes perciben un nivel intermedio a bajo de las vías de comunicación disponibles para entrar a la localidad. Además de niveles bajos en cuanto a la existencia y disponibilidad de sistemas modernos de comunicación (internet, transporte urbano), de infraestructura, equipamiento y servicios para el desarrollo industrial, deficientes 
infraestructuras agropecuarias, así como escasos terrenos o áreas disponibles para desarrollar nuevas empresas o actividades. Al mismo tiempo, perciben como deficientes las acciones de los centros de información que apoyan a los procesos productivos, escaso acompañamiento y apoyo de centros de investigación, especialmente de universidades que apoyen el desarrollo empresarial. Al mismo tiempo, perciben como bajo la existencia de centros de capacitación para el desarrollo (consultoría y formación de recursos humanos. Pese a las debilidades de infraestructura, servicios y accesibilidad de la ciudad a nivel empresarial, los estudiantes consideran, en condiciones intermedias, que la ciudad de Pilar es atractiva para invertir, debido a la buena imagen del municipio. No obstante, las debilidades den ser mejoradas para garantizar el crecimiento y desarrollo de la ciudad; elementos que son consideradas Responsabilidades Sociales básicas derivadas de la función económica: producción, empleo, crecimiento económico (Teixidó, Chavarri, \& Castro, 2002).

Tabla 2. Infraestructura, servicios y accesibilidad

\begin{tabular}{|c|c|c|c|c|c|c|}
\hline & $\begin{array}{l}\text { Nulo/ } \\
\text { inexistente }\end{array}$ & $\begin{array}{l}\text { Muy } \\
\text { Bajo }\end{array}$ & Bajo & $\begin{array}{l}\text { Medio/ } \\
\text { mínimo } \\
\text { aceptable }\end{array}$ & Alto & $\begin{array}{l}\text { Muy } \\
\text { Alto }\end{array}$ \\
\hline $\begin{array}{l}\text { Vías de comunicación en buen estado o } \\
\text { disponibles para entrar a la localidad }\end{array}$ & 3,7 & 5,1 & 24,3 & 43,4 & 20,6 & 2,9 \\
\hline $\begin{array}{l}\text { Existe sistemas modernos de comunicación } \\
\text { (internet, transporte urbano) }\end{array}$ & 3 & 6,7 & 16,3 & 39,3 & 28,1 & 6,7 \\
\hline $\begin{array}{l}\text { Situación de la infraestructura, equipamiento y } \\
\text { servicios para el desarrollo industrial }\end{array}$ & 18,7 & 19,4 & 33,6 & 25,4 & 3 & 0 \\
\hline $\begin{array}{l}\text { Opinión respecto al estado de la infraestructura } \\
\text { agropecuaria }\end{array}$ & 2,9 & 15,4 & 33,8 & 41,9 & 5,1 & 0,7 \\
\hline $\begin{array}{l}\text { existen terrenos o áreas disponibles para } \\
\text { desarrollar nuevas empresas o actividades }\end{array}$ & 0 & 6,6 & 15,4 & 41,9 & 29,4 & 6,6 \\
\hline $\begin{array}{l}\text { Sistemas o centros de información que apoyen a } \\
\text { los procesos productivos }\end{array}$ & 7,4 & 13,3 & 34,8 & 38,5 & 5,2 & 0,7 \\
\hline $\begin{array}{l}\text { Centro de investigación y universidades que } \\
\text { apoyen el desarrollo empresarial }\end{array}$ & 6,7 & 12,6 & 28,1 & 39,3 & 11,9 & 1,5 \\
\hline $\begin{array}{l}\text { Existen centros de capacitación para el desarrollo } \\
\text { (consultoría y formación de recursos humanos }\end{array}$ & 3,7 & 16,2 & 36 & 36 & 6,6 & 1,5 \\
\hline $\begin{array}{l}\text { Servicios especializados que apoyen o capaciten } \\
\text { a las empresas para la producción }\end{array}$ & 4,4 & 19,1 & 27,2 & 32,4 & 15,4 & 1 \\
\hline Es atractiva la ciudad para invertir & 1,5 & 5,9 & 7,4 & 43,4 & 30,9 & 11 \\
\hline Tiene buena imagen el municipio & 5,9 & 8,8 & 10,3 & 51,5 & 18,4 & 5,1 \\
\hline
\end{tabular}

Fuente: elaboración propia.

En relación a la tercera dimensión; Calidad de Vida y RRHH (ver tabla 3), los datos demuestran que los estudiantes perciben que la ciudad de Pilar cuenta mínimamente con los servicios públicos con debilidades en cuanto a la calidad de dichos servicios, misma 
percepción deficiente, refieren respecto a los servicios de trasporte público. Así mismo, perciben escasos niveles de acción de programas y/o proyectos de combate a la pobreza y marginación. Misma percepción (medio a bajo) en relación a la calidad y cobertura de la oferta educativa y de disponibilidad de profesionistas o técnicos capacitados para asesorar a las empresas. También consideran que es muy baja la capacidad en la región para retener a profesionistas o técnicos. Igualmente, los estudiantes perciben que la ciudad escasamente dispone de infraestructura para personas con discapacidad. Así también, desde la perspectiva del estudiante, la ciudad, en niveles intermedio, desarrolla un ambiente cultural y recreativo tanto para las empresas como para sus pobladores.

Tabla 3. Calidad de Vida y de Recursos Humanos

\begin{tabular}{|c|c|c|c|c|c|c|}
\hline & $\begin{array}{l}\text { Nulo/ } \\
\text { inexistente }\end{array}$ & $\begin{array}{l}\text { Muy } \\
\text { Bajo }\end{array}$ & Bajo & $\begin{array}{l}\text { Medio/ } \\
\text { mínimo } \\
\text { aceptable }\end{array}$ & Alto & $\begin{array}{l}\text { Muy } \\
\text { Alto }\end{array}$ \\
\hline $\begin{array}{l}\text { Cuentan con todos los servicios públicos en el } \\
\text { municipio }\end{array}$ & 3 & 11,1 & 15,6 & 50,4 & 13,9 & 19,3 \\
\hline calidad los servicios públicos & 8,1 & 11,8 & 16,2 & 53,7 & 10,3 & 0 \\
\hline $\begin{array}{l}\text { Nivel de cobertura y calidad del servicio del } \\
\text { trasporte urbano }\end{array}$ & 11 & 14 & 22,8 & 43,4 & 8,8 & 0 \\
\hline $\begin{array}{l}\text { Programas, proyectos y acciones de combate a la } \\
\text { pobreza y marginación }\end{array}$ & 21,3 & 23,5 & 31,6 & 19,9 & 3,7 & 0 \\
\hline Calidad y cobertura de la oferta educativa & 5,1 & 17,6 & 23,5 & 42,6 & 11 & 0 \\
\hline $\begin{array}{l}\text { Disponibilidad de profesionistas o técnicos } \\
\text { capacitados para asesorar a las empresas }\end{array}$ & 2,9 & 16,9 & 29,4 & 35,3 & 13,2 & 2,2 \\
\hline $\begin{array}{l}\text { Capacidad en la región para retener a } \\
\text { profesionistas o técnicos }\end{array}$ & 8,1 & 23,5 & 34,6 & 25 & 8,8 & 8,1 \\
\hline $\begin{array}{l}\text { La ciudad cuenta con Infraestructura para } \\
\text { personas con discapacidad }\end{array}$ & 8,1 & 34,6 & 30,1 & 24,3 & 2,9 & 0 \\
\hline $\begin{array}{l}\text { La ciudad desarrolla un Ambiente cultural y } \\
\text { recreativo }\end{array}$ & 5,1 & 10,3 & 16,2 & 39,7 & 25,7 & 2,9 \\
\hline
\end{tabular}

Fuente: elaboración propia.

Referente a la Cohesión social, organización e identidad territorial del municipio de Pilar, los estudiantes universitarios perciben, en niveles mínimos aceptables, actitudes proactivas de la comunidad hacia el desarrollo social y económico, en niveles bajos, la comunidad participa en la toma de decisiones, junto con otros sectores de la sociedad y escasamente existe capacidad de lograr consensos entre los diferentes sectores de la sociedad y débilmente se trabaja en cooperación con los diferentes actores sociales. Estos datos permiten deducir que la ciudad de Pilar presenta debilidades que deberían ser analizados a profundidad, ya que la responsabilidad social concierne a todas las organizaciones y actores sociales, y solo con acciones conjuntas se logra un nivel óptimo de desarrollo sustentable (Vallaeys \& Álvarez Rodríguez, 2019). 
Tabla 4. Cohesión social, organización e identidad territorial

\begin{tabular}{|c|c|c|c|c|c|c|}
\hline & $\begin{array}{l}\text { Nulo/ } \\
\text { inexistente }\end{array}$ & $\begin{array}{l}\text { Muy } \\
\text { Bajo }\end{array}$ & Bajo & $\begin{array}{l}\text { Medio/ } \\
\text { mínimo } \\
\text { aceptable }\end{array}$ & Alto & $\begin{array}{l}\text { Muy } \\
\text { Alto }\end{array}$ \\
\hline $\begin{array}{l}\text { Existe una actitud proactiva de la } \\
\text { comunidad hacia el desarrollo social y } \\
\text { económico }\end{array}$ & 5,2 & 7,4 & 23,7 & 46,7 & 16,3 & 0,7 \\
\hline $\begin{array}{l}\text { Participación comunitaria de todos los } \\
\text { sectores de la sociedad }\end{array}$ & 3,7 & 11,2 & 29,1 & 46,3 & 9,7 & 0 \\
\hline $\begin{array}{l}\text { Condiciones de acuerdos entre los } \\
\text { diferentes actores sociales del municipio } \\
\text { (Capacidad de lograr consensos) }\end{array}$ & 5,1 & 11 & 39,7 & 41,2 & 2,9 & 0 \\
\hline $\begin{array}{l}\text { Cooperación entre los diferentes actores } \\
\text { sociales }\end{array}$ & 2,9 & 12,5 & 41,2 & 39 & 3,7 & 0,7 \\
\hline $\begin{array}{l}\text { Se toman en cuenta a los diferentes grupos } \\
\text { vulnerables para determinar el rumbo del } \\
\text { desarrollo comunitario o municipal }\end{array}$ & 14 & 18,4 & 39,7 & 24,3 & 3,7 & 0 \\
\hline $\begin{array}{l}\text { Grado de organización de la comunidad } \\
\text { para llevar a cabo programas y acciones, } \\
\text { así como gestionar recursos }\end{array}$ & 0,7 & 12,5 & 37,5 & 43,4 & 5,9 & 0 \\
\hline $\begin{array}{l}\text { Liderazgo local (personas o instituciones) } \\
\text { capaz de convocar y movilizar a los } \\
\text { actores sociales en torno al proceso de } \\
\text { desarrollo }\end{array}$ & 4,4 & 11 & 27,2 & 41,9 & 14 & 1,5 \\
\hline $\begin{array}{l}\text { Existe respeto e impulso del patrimonio } \\
\text { histórico y cultural }\end{array}$ & 5,1 & 8,8 & 25 & 39,7 & 20,6 & 0,7 \\
\hline $\begin{array}{l}\text { Grado de vinculación entre centros de } \\
\text { investigación, universidades, sector } \\
\text { productivo y agencias gubernamentales }\end{array}$ & 3,7 & 12,5 & 34,6 & 39 & 8,8 & 1,5 \\
\hline
\end{tabular}

La tabla 5 representa los resultados en forma porcentual de las principales acciones en torno al Medio Ambiente y a los Recursos Naturales, dimensión que muestra niveles relativamente medios respecto al estado del medio ambiente en general de la ciudad, desde la percepción de los estudiantes universitarios. Los estudiantes, quienes poseen una formación de educación superior de base perciben como mínimas a bajas los niveles de educación ambiental (ecología) en la población pilarenses, y deficientes acciones de aprovechamiento y gestión adecuada de recursos naturales de la ciudad por parte de sus pobladores, razón por la que consideran que desde su opinión no se puede garantizar una disponibilidad de agua para el desarrollo a largo plazo, porque los ciudadanos pobremente tienen una cultura y cuidado del agua.

Resalta que los estudiantes universitarios, en bajos niveles, tienen conocimientos sobre la existencia de áreas naturales protegidas en la ciudad, escasamente conocen áreas verdes en la comunidad o tienen algún conocimiento relacionado a la inclusión de criterios ambientales a la producción (armonía entre actividades productivas y medio ambiente). 
Tabla 5. Medio Ambiente y Recursos Naturales

\begin{tabular}{|c|c|c|c|c|c|c|}
\hline & $\begin{array}{l}\text { Nulo/ } \\
\text { inexistente }\end{array}$ & $\begin{array}{l}\text { Muy } \\
\text { Bajo }\end{array}$ & Bajo & $\begin{array}{l}\text { Medio/ } \\
\text { mínimo } \\
\text { aceptabl } \\
\text { e }\end{array}$ & Alto & $\begin{array}{l}\text { Muy } \\
\text { Alto }\end{array}$ \\
\hline $\begin{array}{l}\text { Percepción del estado del medio ambiente } \\
\text { en general de la ciudad }\end{array}$ & 4,5 & 14,9 & 23,9 & 48,5 & 6,7 & 1,5 \\
\hline $\begin{array}{l}\text { Existe cierto Grado de educación ambiental } \\
\text { (ecología) en la población pilarense }\end{array}$ & 10,3 & 19,1 & 27,2 & 39,7 & 3,7 & 0 \\
\hline $\begin{array}{l}\text { Aprovechamiento y gestión adecuada de } \\
\text { recursos naturales de la ciudad }\end{array}$ & 7,4 & 14,7 & 32,4 & 41,2 & 3,7 & 0,7 \\
\hline $\begin{array}{l}\text { Conocimientos de la existencia de áreas } \\
\text { naturales protegidas en la ciudad }\end{array}$ & 20,7 & 17,8 & 28,9 & 28,9 & 3,7 & 0 \\
\hline $\begin{array}{l}\text { Disponibilidad de agua para el desarrollo de } \\
\text { largo plazo }\end{array}$ & 2,2 & 16,9 & 20,6 & 45,6 & 12,5 & 2,2 \\
\hline $\begin{array}{l}\text { Los ciudadanos tienen una cultura y cuidado } \\
\text { del agua }\end{array}$ & 16,9 & 23,5 & 27,2 & 28,7 & 3,7 & 0 \\
\hline $\begin{array}{l}\text { Cocimientos de áreas verdes en la } \\
\text { comunidad }\end{array}$ & 5,9 & 8,1 & 22,2 & 37,8 & 24,4 & 1,5 \\
\hline $\begin{array}{l}\text { Inclusión de criterios ambientales a la } \\
\text { producción (armonía entre actividades } \\
\text { productivas y medio ambiente) }\end{array}$ & 7,4 & 15,6 & 34,1 & 37 & 5,2 & 0,7 \\
\hline
\end{tabular}

Fuente: elaboración propia.

Siguiendo con la dimensión Organización, Normatividad y Programas de ordenamiento ecológico y territorial, se vuelve a evidenciar percepciones débiles sobre RSE y DS en los estudiantes. Los datos observados en la tabla 6 muestran una tendencia media/mínima aceptable en la mayoría de sus indicadores. Esta variable coincide con los niveles medio (decreciendo a niveles bajos y muy bajos) presentados en las otras variables citadas más arriba, lo que hace deducir que el municipio, en su conjunto de habitantes, demuestran incipientes acciones que despierten una actitud reflexiva en los estudiantes en relación al medio ambiente, lo económico y lo social; como factores que se interrelacionan para crear un desarrollo social responsable, sustentable y sostenible con el tiempo (Vallaeys \& Álvarez Rodríguez, 2019).

Tabla 6. Organización, Normatividad y Programas de ordenamiento ecológico y territorial

\begin{tabular}{|c|c|c|c|c|c|c|}
\hline & $\begin{array}{l}\text { Nulo/ } \\
\text { inexistente }\end{array}$ & $\begin{array}{l}\text { Muy } \\
\text { Bajo }\end{array}$ & Bajo & $\begin{array}{l}\text { Medio/ } \\
\text { mínimo } \\
\text { aceptable }\end{array}$ & Alto & $\begin{array}{l}\text { Muy } \\
\text { Alto }\end{array}$ \\
\hline $\begin{array}{l}\text { Existencia/contribución de organizaciones } \\
\text { sociales que protejan al medio ambiente }\end{array}$ & 6,6 & 16,2 & 35,3 & 33,1 & 8,8 & 0 \\
\hline $\begin{array}{l}\text { Existe grupos de investigación sobre desarrollo } \\
\text { ambiental }\end{array}$ & 15,4 & 16,9 & 33,8 & 28,7 & 5,1 & 0 \\
\hline $\begin{array}{l}\text { Existe infraestructura ambiental (plantas de } \\
\text { tratamiento de agua; disposición de desechos, } \\
\text { entro otros en la ciudad }\end{array}$ & 8,2 & 21,6 & 25,4 & 40,3 & 4,5 & 0 \\
\hline
\end{tabular}




\begin{tabular}{|c|c|c|c|c|c|c|}
\hline $\begin{array}{l}\text { Se aplica la normatividad ambiental en la } \\
\text { ciudad }\end{array}$ & 9,6 & 20,7 & 36,3 & 31,1 & 2,2 & 0 \\
\hline $\begin{array}{l}\text { Se implementan programas de ordenamiento } \\
\text { ecológico-territorial en la ciudad }\end{array}$ & 11,8 & 19,1 & 33,8 & 29,4 & 4,4 & 4,4 \\
\hline
\end{tabular}

\section{Conclusiones}

Los resultados del estudio permiten concluir que la ciudad de Pilar, capital del departamento de Neembucú, en Paraguay, desarrolla un nivel intermedio de Responsabilidad Social y Desarrollo Sustentable, desde la percepción de los estudiantes universitarios. Dicho nivel se traduce en acciones mínimas aceptables de desarrollo, por tal razón, el panorama actual de la ciudad de Pilar, desde las primeras impresiones de los estudiantes universitarios, motiva a abordar mejoras en cuanto a las seis dimensiones que propone Wong (2005) estructura económica, agentes económicos e innovación, Infraestructura, servicios y accesibilidad, Calidad de vida y RRHH, Cohesión social, organizaciones e identidad territorial, Medio ambiente y Recursos Naturales y Organizaciones, Normatividad y Programas de ordenamiento ecológico y territorial, ya que en ninguna de las dimensiones citadas se han evidenciado indicadores en condiciones de fortalezas, por el contrario, todas la variables presentan debilidades que deben de ser potenciados para elevar la percepción de los jóvenes a niveles que permitan, del ejemplo del municipio, despertar una actitud reflexiva y fomentar a través de ellas, la adopción de acciones socialmente responsables y sustentables, desde el contexto educativouniversitario, ya que el estudiantado conforma un actor social clave con capacidad de influir en el debate público (Gallardo-Milanés, et.al. 2019).

\section{Reflexiones finales}

La importancia del estudio sobre la percepción de los estudiantes universitarios, específicamente de los alumnos de la carrera de Licenciatura en Contaduría Público Nacional, dependiente de la Facultad de Ciencias Contables, Administrativas y Económicas de la Universidad Nacional de Pilar, Neembucú, Paraguay, obedece a la necesidad de conocer como han interpretado las interrogantes sobre las dimensiones que mide la Responsabilidad Social y Desarrollo Sustentable, como una manera de relacionar la teoría con la práctica, ya que se han desarrollados contenidos relacionados a RS y DS alineadas a la misión principal de dicha institución y en relación al perfil de egreso de la carrera que busca «Formar profesionales con sólidos conocimientos en las Ciencias 
Contables; capaces de emprender, asesorar y gerenciar entidades; basados en principios éticos, con compromiso social, en la búsqueda del desarrollo sustentable y del mejoramiento de la calidad de vida de la población a nivel local y nacional» (FCCAYE.UNP, 2020).

\section{LISTA DE REFERENCIAS}

Gándara Mendoza, A., \& Villafán Vidales, K. B. (2019). CONTRIBUCIONES DE LA TEORÍA DE REDES HACIA LA SUSTENTABILIDAD. Revista de la Facultad de Contaduría y Ciencias Administrativas, 114-129. Obtenido de http://rfcca.umich.mx/index.php/rfcca/article/view/125

Barrios, N. M., \& Nuñez Esquivel, G. (2019). RESPONSABILIDAD SOCIAL UNIVERSITARIA: PERCEPCION DE LOS ESTUDIANTES HACIA ESTA REALIDAD EN PRÁCTICA. INPEAU/UFSC. Obtenido de https://repositorio.ufsc.br/handle/123456789/201878

Coppari, N., Bagnoli, L., \& Codas, G. (2016). PERCEPCIÓN DE LA RESPONSABILIDAD SOCIAL UNIVERSITARIA EN ESTUDIANTES Y DOCENTES UNIVERSITARIOS PARAGUAYOS: UN ESTUDIO PILOTO. XVI COLOQUIO INTERNACIONAL EN GESTIÓN UNIVERSITARIA EN AMERICA. Gestion de la Investigacion y Compromiso Social de la Universidad. Obtenido de https://www.utic.edu.py/repositorio/COLOQUIOSSIMPOSIOS/COLOQUIOS/XVI\%20Coloquio\%20Internacional/7.\%20Norma $\%$ 20Copary\%20RSU\%20Py.pdf

Coraggio, J. L. (2002). Universidad y desarrollo local. Ponencia presentada en el Seminario Internacional iLa educacî̂n superior y las nuevas tendencias" (págs. 1-21). Quito: Consejo Nacional de EducaciÛn Superior (CONESUP).

FCCAYE.UNP. (2020). Visión, Misiones, Valores y Perfil de egreso d ela carrera de Contaduría Pública Nacional. Pilar. Neembucu, Paraguay: UNP.

Fernández, L., \& Gutiérrez, M. (2013). Bienestar Social, Económico y Ambiental para las Presentes y Futuras Generaciones. Información Tecnológica Vol 24, 121 130. doi:doi: 10.4067/S0718-07642013000200013

Fonseca Ferís, R. (2020). esponsabilidad social universitaria. . Arandu-UTIC. Revista Científica Internacional De La Universidad Tecnológica Intercontinental, , 179192. 
Gallardo-Milanés, O. A., Olivera-Pátaro, C. S., \& Mezzomo, F. A. (2019). El estudiantado universitario de UNESPAR-Brasil: sus percepciones sobre el desarrollo sostenible. Revista Educación. Obtenido de http://www.redalyc.org/articulo.oa?id=44057415033

Gallardo-Milanés, O., Satiê de Olivera-Pátaro, C., \& Mezzomo, F. (2019). El estudiantado universitario de UNESPAR-Brasil: sus percepciones sobre el desarrollo sostenible. Revista Educación vol.43 n.l San José, San Pedro, Montes de Oca, 327-343.

Galván, A., Miranda, J., Baéz, M., Acosta, E., \& Murillo, C. (2014). Análisis de fiabilidad de un instrumento para medir la percepción del nivel de desarrollo sustentable de Navojoa, Sonora. Revista Iberoamericana para la Investigación y el Desarrollo Educativo ISSN 2007 - 7467, 1-12.

Hernandez Sampieri, R., Fernandez Collado, C., \& Pilar Batista , L. (2014). Metodologia de la investigación. México: McGRAW-HILL / INTERAMERICANA EDITORES, S.A. DE C.V.

Litzner Ordóñez, L. I., \& Werner RIEß. (2019). LA EDUCACIÓN PARA EL DESARROLLO SOSTENIBLE EN LA UNIVERSIDAD BOLIVIANA. PERCEPCIONES DEL PROFESORADO. Teoría de la Educación. Revista Interuniversitaria, 149-173. Obtenido de https://revistas.usal.es/index.php/11303743/article/view/19037

Lopez Paniagua, R., Ayala Ortiz, D., \& Arellanes Cancino, Y. (2017). Gobernabilidad Democratica y Desarrollo Sutentable. Economía y Sociedad, vol. XXI, núm. 36,, $61-75$.

López Regalado, M. E., \& Ahumada Tello, E. (2018). Percepción de estudiantes sobre la Responsabilidad Social Universitaria. La Facultad de Contaduría y Administración en UABC Tijuana. Revista Argentina de Investigación en Negocios, 23-39. Obtenido de http://ppct.caicyt.gov.ar/rain/article/view/v4n1a03

Martínez Castillo, R., \& Martínez Chaves, D. (2016). Perspectivas de la sustentabilidad: teoría y campos de análisis. Revista Pensamiento Actual, 123-145. Obtenido de https://dialnet.unirioja.es/servlet/articulo?codigo $=5821458$

Maruri Avidal, C., \& Torres Rivera, A. D. (2019). Gestión de la sustentabilidad en las cadenas de suministro: Un acercamiento desde la teoría. Red Internacional de Investigadores en Competitividad, 1523-1539. Obtenido de https://riico.net/index.php/riico/article/view/1868/1627 
ONU Organización de la Naciones Unidas. (2016). Agenda 2030 y los Objetivos de Desarrollo Sostenible. Una oportunidad para América Latina y el Caribe. CEPAL.

Palomino-Garcia, V. (2020). Programa de educación ambiental y la responsabilidad social universitaria de los estudiantes de la Universidad Peruana Los Andes. Repositorio UPLA, 215.

Parra Melgarejo, P. (2018). Percepción de la responsabilidad socialmunicipal en trabajadores de la Municipalidad. Huancayo - Perú.: Tesis para optar el Grado Académico de Maestro en Responsabilidad Social, Relaciones Comunitarias y Gestión de Conflictos).

Perez Colina, E. (2020). Responsabilidad social universitaria desde la percepción de los estudiantes en la Escuela de Postgrado de La UNPRG de Lambayeque (Tesis de pregrado). Universidad Católica Santo Toribio de Mogrovejo, Chiclayo, Perú. Obtenido de http://hdl.handle.net/20.500.12423/2555

Pérez-García, A. (2017). LA RESPONSABILIDAD SOCIAL CORPORATIVA DESDE EL ENFOQUE DE LASRELACIONES PÚBLICAS: ESTRATEGIA DE GESTIÓN RELACIONAL EN ELONTEXTO DEL DESARROLLO LOCAL Y TURISMO SOSTENIBLE. HOLOS, vol. 7 Instituto Federal de Educação, Ciência e Tecnologia do Rio Grande do Norte, 183-197.

Quinche Martín, F. L. (2017). Una mirada crítica a las teorías predominantes de la responsabilidad social corporativa. Revista Facultad de Ciencias Económicas: Investigación y Reflexión, 159-178. Obtenido de http://dx.doi.org/10.18359/rfce.3071

Rodrigo-Cano, D., Picó, M. J., \& Dimuro, G. (2019). Los Objetivos de Desarrollo Sostenible como marco para la acción y la intervención social y ambiental. Retos. Revista de Ciencias de la Administración y Economía, 25-36. Obtenido de https://doi.org/10.17163/ret.n17.2019.02

Teixidó, S., Chavarri, R., \& Castro, A. (2002). Responsabilidad Social Empresarial en Chile: Perspectivas para una matriz de análisis. Santiago, Chile: Fundación PROhumana,.

Vallaeys, F., \& Álvarez Rodríguez, J. (2019). HACIA UNA DEFINICIÓN

LATINOAMERICANA DE RESPONSABILIDAD SOCIAL UNIVERSITARIA. APROXIMACIÓN A LAS PREFERENCIAS CONCEPTUALES DE LOS UNIVERSITARIOS. Educación XX1, 93-116. doi: 10.5944/educXX1.19442 
Wong, P. (2005). Propuesta metodológica para el análisis estratégico participativo de desarrollo local-regional. México: Centro de Investigación en Alimentación y Desarrollo, A.C. (CIAD, A.C.). 